\title{
Relations of a planar domains bounded by hyperbolas with families of holomorphic functions
}

\section{S. Kanas ${ }^{1 *}$ (D) V.S. Masih² (1D and A. Ebadian³}

$\overline{{ }^{*} \text { Correspondence: skanas@ur.edu.pl }}$ ${ }^{1}$ University of Rzeszow, Rzeszów, Poland

Full list of author information is available at the end of the article

\begin{abstract}
We consider a family of analytic and normalized functions with the property that $z f^{\prime}(z) / f(z)$ (or $\left.1+z f^{\prime \prime}(z) / f^{\prime}(z)\right)$ lies in a domain bounded by a right branch of a hyperbola $\rho=\rho(s)=\left(2 \cos \frac{\varphi}{s}\right)^{-s}$, where $0<s \leq 1$ and $|\varphi|<(\pi s) / 2$. A comprehensive characteristic of that families and relations with the well known families of univalent functions are presented. Some relevant examples are indicated.
\end{abstract}

MSC: $30 C 45 ; 30 C 80$

Keywords: Univalent functions; Subordination; Starlike and convex functions; Domain bounded by hyperbola

\section{Introduction and motivation}

Let $\mathcal{H}$ denote the class of functions $f$ holomorphic in the open unit disc $\mathbb{D}=\{z \in \mathbb{C}:|z|<1\}$ of the complex plane $\mathbb{C}$, and with the power series

$$
f(z)=z+\sum_{n=2}^{\infty} a_{n} z^{n} \quad(z \in \mathbb{D}) .
$$

By $\mathcal{S}$ we denote the subclass of $\mathcal{H}$ consisting of univalent functions, and by $\mathcal{S T}, \mathcal{C} \mathcal{V}$ the best known subclasses of $\mathcal{S}$ containing functions starlike with respect to the origin and convex, respectively. Their geometric properties and various applications have caused rapid development of research in these directions. Many its subclasses have been introduced and investigated. The main feature of the new definitions is usually fact that either $z f^{\prime}(z) / f(z)$ or $1+z f^{\prime \prime}(z) / f^{\prime}(z)$ is located in a some region contained in a right halfplane. A uniform approach to such definitions was proposed by Ma and Minda [7]. They introduced a special class of functions $\varphi$ that map the unit disk onto the domains $\varphi(\mathbb{D})$ symmetric with respect to the real axis, starlike with respect to $\varphi(0)=1$, and with $\varphi^{\prime}(0)>0$. A family $\mathcal{P}(\varphi)$ that consists of holomorphic functions $p$ such that $p(0)=1$ and $p(\mathbb{D}) \subset \varphi(\mathbb{D})$ (or in terms of subordination as $p \prec \varphi$ ) have also been defined in [7] (in connection with the function $\varphi)$. The Carathéodory class $\mathcal{P}=\left\{p(z)=1+p_{1} z+p_{2} z^{2}+\cdots, \Re p(z)>0, z \in \mathbb{D}\right\}$ is simply the class $\mathcal{P}((1+z) /(1-z))$. The class of starlike univalent functions $\mathcal{S T}$ (and convex functions $\mathcal{C V}$, resp.) can be described as $z f^{\prime} \mid f \in \mathcal{P}((1+z) /(1-z))\left(\right.$ or $1+z f^{\prime \prime} \mid f^{\prime} \in \mathcal{P}((1+z) /(1-z))$,

(c) The Author(s) 2019. This article is distributed under the terms of the Creative Commons Attribution 4.0 International License (http://creativecommons.org/licenses/by/4.0/), which permits unrestricted use, distribution, and reproduction in any medium, provided you give appropriate credit to the original author(s) and the source, provide a link to the Creative Commons license, and indicate if changes were made. 
resp.). It is seen that if $\varphi$ varies, $\mathcal{P}(\varphi)$ generates a number of well known subclasses of the Carathéodory class. In a such way, various similar subclasses of $\mathcal{P}$ were defined and studied through a years. Leading examples of $\mathcal{P}$ are $\mathcal{P}(\alpha)=\mathcal{P}\left(\varphi_{\alpha}(z)\right)=\mathcal{P}((1+(1-2 \alpha) z) /(1-z))$, $\mathcal{P}_{\gamma}=\mathcal{P}\left(\varphi^{\gamma}(z)\right)=\mathcal{P}\left(((1+z) /(1-z))^{\gamma}\right), \mathcal{P}(A, B)=\mathcal{P}\left(\varphi_{A, B}(z)\right)=\mathcal{P}((1+A z) /(1+B z))$, etc. Related families of starlike and convex functions of order $\alpha(0 \leq \alpha<1)$ (strongly starlike and convex of order $\gamma(0<\gamma \leq 1)$, resp.) were defined as follows:

$$
\begin{aligned}
& \mathcal{S T}(\alpha)=\left\{f \in \mathcal{H}: z f^{\prime} \mid f \prec \varphi_{\alpha} \text { in } \mathbb{D}\right\}=\left\{f \in \mathcal{H}: \Re \frac{z f^{\prime}(z)}{f(z)}>\alpha, z \in \mathbb{D}\right\}, \\
& \mathcal{S T}_{\gamma}=\left\{f \in \mathcal{H}: z f^{\prime} \mid f \prec \varphi^{\gamma} \text { in } \mathbb{D}\right\}=\left\{f \in \mathcal{H}:\left|\operatorname{Arg}\left\{\frac{z f^{\prime}(z)}{f(z)}\right\}\right|<\frac{\gamma \pi}{2}, z \in \mathbb{D}\right\},
\end{aligned}
$$

and also the corresponding subclasses $\mathcal{C} V(\alpha), \mathcal{C} \mathcal{V}_{\gamma}$ of convex functions, for which $1+$ $z f^{\prime \prime} \mid f^{\prime} \prec \varphi_{\alpha}$ or $1+z f^{\prime \prime} \mid f^{\prime} \prec \varphi^{\gamma}(z)$, respectively (see, for example $[1,9,11]$ ). We note that $\mathcal{S T}=\mathcal{S} \mathcal{T}(0)=\mathcal{S} \mathcal{T}_{1}$ and $\mathcal{C V}=\mathcal{C} \mathcal{V}(0)=\mathcal{C} \mathcal{V}_{1}$

An interesting families of the domains that are bounded by a conic sections were introduced and studied in $[5,6]$. Such domains are related to $k$-starlike functions $k-\mathcal{S T}$, and $k$-uniformly convex function, denoted $k-\mathcal{U C V}$. A univalent function $f \in k-\mathcal{S T}$ if the expression $z f^{\prime} / f$ satisfies

$$
\Re \frac{z f^{\prime}(z)}{f(z)}>k\left|\frac{z f^{\prime}(z)}{f(z)}-1\right| \quad(z \in \mathbb{D}),
$$

and $f \in k-\mathcal{U C V}$ if $\Re\left(1+z f^{\prime \prime}(z) / f^{\prime}(z)\right)>k\left|z f^{\prime \prime}(z) / f^{\prime}(z)\right|$ for $z \in \mathbb{D}$. Equivalently, these families may be geometrically described by the fact that the expression $p(z)=z f^{\prime}(z) / f(z)($ or $p(z)=$ $1+z f^{\prime \prime}(z) / f^{\prime}(z)$, resp.) lies in a domain $\Omega_{k}$ for $z \in \mathbb{D}$, where

$$
\Omega_{k}=\left\{u+\mathrm{i} v: u^{2}>k^{2}(u-1)^{2}+k^{2} v^{2}\right\} .
$$

We note that $\Omega_{k}$ is bounded by the conic sections of a shape depending on a parameter $k$ and contained in a right halfplane. $\partial \Omega_{k}$ reduces to the imaginary axis for $k=0$, a hyperbola for $0<k<1$, parabola for $k=1$, and finally to an ellipse, when $1<k<\infty$. It was observed in $[5,6]$ that each $\Omega_{k}$ is symmetric with respect to the real axis and starlike with respect to the point 1 . Additionally the functions $p_{k}$, constructed in [6], see also [4], that map the unit disk onto $\Omega_{k}$ satisfy $p_{k}(0)=1, p_{k}^{\prime}(0)>0$, for $0 \leq k<\infty$, therefore fulfill the Ma and Minda assertions.

We also recall that the hyperbola considered in $[5,6]$ is given by

$$
\partial \Omega_{k}=\left\{u+\mathrm{i} v: u^{2}=k^{2}(u-1)^{2}+k^{2} v^{2}, u>0\right\}
$$

and corresponds to the parameter $0<k<1$. Moreover, the points of intersection of $\partial \Omega_{k}$ with the real axis is $(u, 0)=(k /(k+1), 0)$ and the slope angle of asymptotes of $\partial \Omega_{k}$ to the real axis equals $\arctan (1 / k)$.

This work is intended as an attempt at defining a new subfamily of $\mathcal{P}$ related to the domains bounded by a right branch of a hyperbolas $H(s)$ for $0<s \leq 1$, where

$$
H(s)=\left\{\rho \mathrm{e}^{\mathrm{i} \varphi}: \rho=\frac{1}{\left(2 \cos \frac{\varphi}{s}\right)^{s}},-\frac{\pi s}{2}<\varphi<\frac{\pi s}{2}\right\} .
$$


It is easy to observe that the intersection point of $H(s)$ and real axis is $(u, 0)=\left(1 / 2^{s}, 0\right)$ and the slope angle to the real axis is equal $(\pi s) / 2$. Taking into account these facts, we want to emphasize then the fact that none hyperbola $\partial \Omega_{k}$ reduces to $H(s)$ and conversely. Indeed, considering a location of common points of the hyperbola $H(s)$, hyperbola $\partial \Omega_{k}$ with the real axis and, we obtain the relation $k /(k+1)=1 / 2^{s}$ that holds for $k=1 /\left(2^{s}-1\right)>1$. But when $k>1$ the $\partial \Omega_{k}$ is the ellipse.

It is of interest to study the common relations between $H(s)$ and $\partial \Omega_{k}$ for any $0 \leq k<\infty$ and $0<s \leq 1$. The detailed investigation of geometric behavior of $\partial \Omega_{k}$ and $H(s)$ provides a following relations:

$$
\begin{aligned}
& H(s) \cap \partial \Omega_{k}=\emptyset \quad \text { for } 0 \leq k \leq 1 / 2^{s} \text { and } k>1 /\left(2^{s}-1\right), 0<s \leq 1, \\
& H(s) \cap \partial \Omega_{k}=\{M\} \quad \text { for } k=1 /\left(2^{s}-1\right), 0<s \leq 1, \text { where } M=\left(1 / 2^{s}, 0\right), \\
& H(s) \cap \partial \Omega_{k}=\left\{M_{1}, M_{2}\right\} \quad \text { for } 1 / 2^{s}<k<1 /\left(2^{s}-1\right), 0<s \leq 1,
\end{aligned}
$$

where

$$
M_{1,2}=\left(\frac{k 2^{s-1}}{k 2^{s}-1}, \pm \frac{\sqrt{\left(1-k^{2}\right) 4^{s-1}+k 2^{s}-1}}{k 2^{s}-1}\right) .
$$

For the case $0 \leq k \leq 1 / 2^{s}$ a domain bounded by $H(s)$ is contained in a domain bounded by $\partial \Omega_{k}$, and for $k \geq 1 /\left(2^{s}-1\right)$ conversely, the ellipse bounded by $\partial \Omega_{k}$ is contained in a domain bounded by a hyperbola $H(s)$. Let us denote by $\mathbb{H}(s)$ a domain bounded by hyperbola $H(s)$. Thus, we have the following inclusion:

$$
\begin{array}{ll}
\mathbb{H}(s) \subset \Omega_{k} & \text { for } 0 \leq k \leq 1 / 2^{s}, 0<s \leq 1 \\
\Omega_{k} \subset \mathbb{H}(s) & \text { for } k \geq 1 /\left(2^{s}-1\right), 0<s \leq 1 .
\end{array}
$$

In the remaining cases of $k$ the considered domains have nonempty intersection set (see Fig. 1). These observation will have significant consequences for the further relations between corresponding classes of analytic functions, but also means that the domains bounded by hyperbolas $H(s)(0<s \leq 1)$ are completely different from the similar domains bounded by the hyperbola $\partial \Omega_{k}$.

We also note the obvious difference between $\mathbb{H}(s)$ and other domains contained in a right halfplane: disks, halfplanes and angular domains, that have appeared in the investigations of various subclasses of analytic functions.

From the above reasons we wish to find an analytic function that map the unit disk onto a domain bounded by the hyperbola $H(s)$, define and investigate a subfamily of $\mathcal{P}$ related to those domains. As a natural extension of that investigation we will define a subfamily $\mathcal{S} \mathcal{T}_{\text {hpl }}(s)$ of the starlike functions for which $z f^{\prime} / f$ is contained in a domain bounded by the hyperbola $H(s)$ (and related class $\mathcal{C} \mathcal{V}_{\text {hpl }}(s)$ of convex functions). It is also natural to try to give a sufficient condition for the function $f \in \mathcal{H}$ to be a member of the class $\mathcal{S} \mathcal{T}_{\text {hpl }}(s)$ (or $\mathcal{C} \mathcal{V}_{\text {hpl }}(s)$, resp.) and solve several extremal problems.

In order to achieve our aim we recall some definitions and preliminary results from the geometric theory of analytic functions. By $\mathcal{B}$ we denote the class of analytic self-mappings 


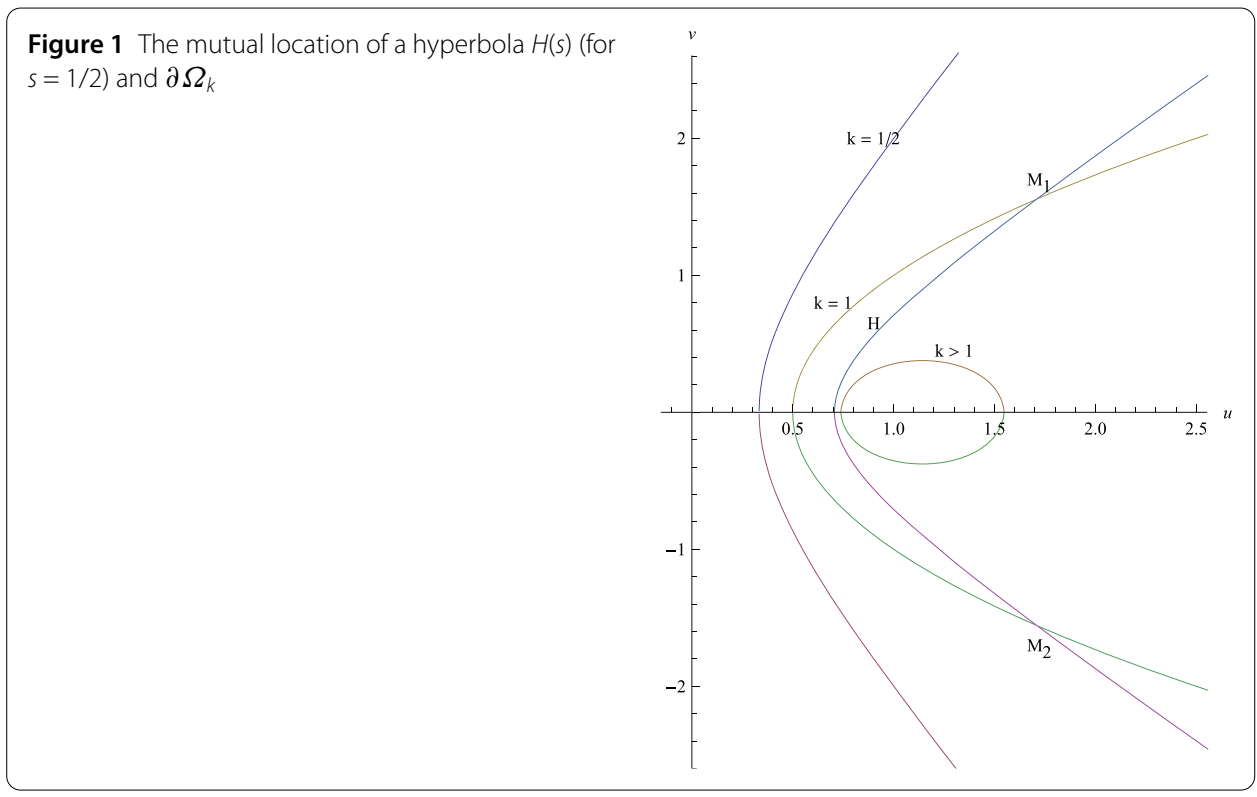

of the unit disk that send the origin onto the origin, i.e.

$$
\mathcal{B}=\{\omega(z) \in \mathcal{H}, \omega(0)=0,|\omega(z)|<1, z \in \mathbb{D}\} .
$$

The class $\mathcal{B}$ is known as the class of Schwarz functions.

We say that $f$ is subordinate to $F$ in $\mathbb{D}$, written $f(z) \prec F(z)$ (or $f \prec F$ ), if there exists a function $\omega \in \mathcal{B}$ such that $f(z)=F(\omega(z))(z \in \mathbb{D})$ see, for example [2,3]. From that definition it is easy to show that if $F$ is univalent in $\mathbb{D}$ then $f \prec F$ holds if and only, if $f(0)=F(0)$, and $f(\mathbb{D}) \subset F(\mathbb{D})$.

Let us denote by $\mathcal{Q}$ the class of functions $f$ that are analytic and injective on $\overline{\mathbb{D}} \backslash E(f)$, where

$$
E(f)=\left\{\zeta: \zeta \in \partial \mathbb{D} \text { and } \lim _{z \rightarrow \zeta} f(z)=\infty\right\}
$$

and that are such that

$$
f^{\prime}(\zeta) \neq 0 \quad \text { for } \zeta \in \partial \mathbb{D} \backslash E(f)
$$

Lemma 1.1 ([8]) Let $q \in \mathcal{Q}$ with $q(0)=1$ and let $p(z)=1+p_{1} z+\cdots$ be analytic in $\mathbb{D}$ with $p(z) \neq 1$. If $p \nprec q$ in $\mathbb{D}$ then there exist points $z_{0} \in \mathbb{D}$ and $\zeta \in \partial \mathbb{D} \backslash E(q)$ and there exist a real number $m \geq 1$ for which

$$
p\left(|z|<\left|z_{0}\right|\right) \subset q(\mathbb{D}), \quad p\left(z_{0}\right)=q(\zeta), \quad z_{0} p^{\prime}\left(z_{0}\right)=m \zeta q^{\prime}(\zeta) .
$$

\section{The class $\mathcal{P}\left(\mathfrak{q}_{s}\right)$ and its properties}

This section provides a detailed exposition of an analytic function that maps the unit disk onto a domain bounded by a hyperbola $H(s)$ and contained in a right halfplane. Let

$$
\mathfrak{q}_{s}(z):=\frac{1}{(1-z)^{s}}=\mathrm{e}^{-s \log (1-z)} \quad(0<s \leq 1, z \in \mathbb{D}),
$$


where the branch of the logarithm is determined by $\mathfrak{q}_{s}(0)=1$. It is easy to check that

$$
\begin{aligned}
\mathfrak{q}_{s}(z) & =1+\sum_{n=1}^{\infty} q_{n} z^{n}=1+\sum_{n=1}^{\infty} \frac{s(s+1) \cdots(s+n-1)}{n !} z^{n} \\
& =1+s z+\frac{s(s+1)}{2} z^{2}+\frac{s(s+1)(s+2)}{6} z^{3}+\cdots \quad(z \in \mathbb{D}) .
\end{aligned}
$$

The function $\mathfrak{q}_{s}$ maps the unit disk onto a domain bounded by a right branch of a hyperbola

$$
H(s)=\left\{\rho \mathrm{e}^{\mathrm{i} \varphi}: \rho=\frac{1}{\left(2 \cos \frac{\varphi}{2}\right)^{s}},-\frac{\pi s}{2}<\varphi<\frac{\pi s}{2}\right\} .
$$

Indeed, setting $z=\mathrm{e}^{\mathrm{i} \theta}(\theta \in(0,2 \pi))$ we obtain

$$
\rho \mathrm{e}^{\mathrm{i} \varphi}=\mathfrak{q}_{s}\left(\mathrm{e}^{\mathrm{i} \theta}\right)=\frac{1}{\left(1-\mathrm{e}^{\mathrm{i} \theta}\right)^{s}}=\mathrm{e}^{-s \log \left|1-\mathrm{e}^{\mathrm{i} \theta}\right|-s \operatorname{Arg}\left(1-\mathrm{e}^{\mathrm{i} \theta}\right)}=\frac{\mathrm{e}^{\mathrm{i} \frac{\pi-\theta}{2} s}}{\left(2 \sin \frac{\theta}{2}\right)^{s}} .
$$

Thus, we have

$$
\begin{aligned}
& \Re \mathfrak{q}_{s}\left(\mathrm{e}^{\mathrm{i} \theta}\right)=\frac{\cos \left(\frac{\pi-\theta}{2} s\right)}{\left(2 \sin \frac{\theta}{2}\right)^{s}}, \quad \Im \mathfrak{q}_{s}\left(\mathrm{e}^{\mathrm{i} \theta}\right)=\frac{\sin \left(\frac{\pi-\theta}{2} s\right)}{\left(2 \sin \frac{\theta}{2}\right)^{s}}, \\
& \rho=\frac{1}{\left(2 \sin \frac{\theta}{2}\right)^{s}}, \quad \text { with } \varphi=\frac{\pi-\theta}{2} s .
\end{aligned}
$$

Setting $\varphi=(\theta-\pi) s / 2$, after simple computation, we obtain

$$
\rho=\frac{1}{\left(2 \cos \frac{\varphi}{s}\right)^{s}} \quad \text { with }\left(-\frac{\pi s}{2}<\varphi<\frac{\pi s}{2}\right)
$$

The function

$$
g(\theta)=\Re \mathfrak{q}_{s}\left(\mathrm{e}^{\mathrm{i} \theta}\right)=\frac{\cos \left(\frac{\pi-\theta}{2} s\right)}{\left(2 \sin \frac{\theta}{2}\right)^{s}}
$$

attains its minimal value when $\theta=\pi$, i.e., we have

$$
\Re \mathfrak{q}_{s}\left(\mathrm{e}^{\mathrm{i} \theta}\right) \geq \frac{1}{2^{s}} \quad(0<\theta<2 \pi) .
$$

Also, we note that $\Re \mathfrak{q}_{s}\left(\mathrm{e}^{\mathrm{i} \theta}\right) \rightarrow \infty$ for $\theta \rightarrow 0$. From (2.1) we obtain that the boundary $\mathfrak{q}_{s}\left(\mathrm{e}^{\mathrm{i} \theta}\right)$ satisfies the equality $1 / \rho=\left(2 \sin \frac{\theta}{2}\right)^{s}=\left(2 \cos \frac{\varphi}{s}\right)^{s}$. Additionally, we have $\mathfrak{q}_{s}(0)=1$, therefore a right branch of a hyperbola

$$
H(s)=\left\{\rho \mathrm{e}^{\mathrm{i} \varphi} \in \mathbb{C}: \rho=\frac{1}{\left(2 \cos \frac{\theta}{s}\right)^{s}},|\varphi|<\frac{\pi s}{2}\right\}
$$

is a boundary of a domain $\mathfrak{q}_{s}(\mathbb{D})$, which we denote by $\mathbb{H}(s)$. The domain $\mathbb{H}(s)$ is symmetric about the real axis, starlike with respect to the point $\mathfrak{q}_{s}(0)=1$, and it satisfies $\mathfrak{q}_{s}^{\prime}(0)=s>0$. Also, the hyperbola $H(s)=\partial \mathbb{H}(s)$ has a vertex at $\left(2^{-s}, 0\right)$ and the slant asymptotes $v= \pm \tan (\pi s / 2) u$ (see Fig. 2). 


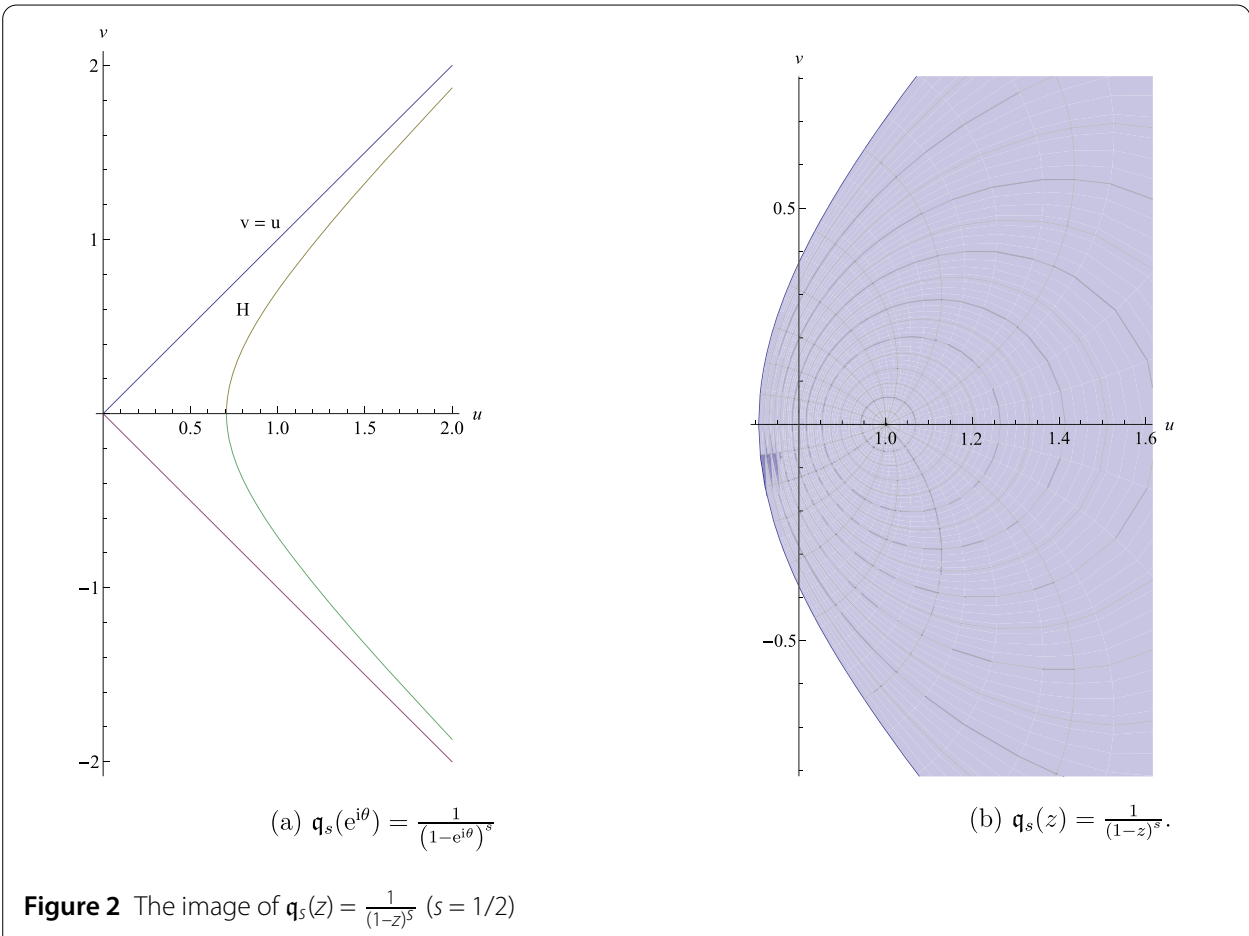

A special and interesting case of $H(s)$ is that for $s=1 / 2$. We then obtain $\mathfrak{q}_{1 / 2}(z)=\frac{1}{\sqrt{1-z}}$, and the hyperbola reduces to that given by the relation

$$
H(1 / 2)=\left\{u+\mathrm{i} v: u^{2}-v^{2}=1 / 2, u>0\right\} .
$$

Lemma 2.1 The function $\mathfrak{q}_{s}$ is convex univalent in $\mathbb{D}$, moreover $g(z)=\left(\mathfrak{q}_{s}(z)-1\right) / s \in$ $\mathcal{C V}((1-s) / 2)$. Also, if $|z|=r<1$, then

$$
\min _{|z|=r}\left|\mathfrak{q}_{s}(z)\right|=\mathfrak{q}_{s}(-r)=\frac{1}{(1+r)^{s}} \quad \text { and } \quad \max _{|z|=r}\left|\mathfrak{q}_{s}(z)\right|=\mathfrak{q}_{s}(r)=\frac{1}{(1-r)^{s}}
$$

Proof We first observe that $\mathfrak{q}_{s}$ is convex by a convexity property of a set $\mathfrak{q}_{s}(\mathbb{D})$. To deduce the univalence, we use the results on univalence of some integrals from [10]. In order to prove (2.2) we consider the function $Q(\theta)$ for $\theta \in[0,2 \pi)$, where

$$
Q(\theta)=\left|\mathfrak{q}_{s}\left(r \mathrm{e}^{\mathrm{i} \theta}\right)\right|=\frac{1}{\left|1-r \mathrm{e}^{\mathrm{i} \theta}\right|^{s}}=\frac{1}{\left(1+r^{2}-2 r \cos \theta\right)^{s / 2}} \quad(0<r<1) .
$$

Since $Q(\theta)$ attains its minimum at $\theta=\pi$ and maximum at $\theta=0$, (2.2) follows.

Let us consider $g(z)=\left(\mathfrak{q}_{s}(z)-1\right) / s(z \in \mathbb{D})$. Then we have

$$
\Re\left(1+\frac{z g^{\prime \prime}(z)}{g^{\prime}(z)}\right)=\Re\left(\frac{1+s z}{1-z}\right)>\frac{1-s}{2},
$$

so that $g \in \mathcal{C} \mathcal{V}((1-s) / 2)$, as asserted. 
Definition 2.2 By $\mathcal{P}\left(\mathfrak{q}_{s}\right)$ we denote a class of all analytic functions $p$ such that $p(0)=1$ and $p(z) \prec \mathfrak{q}_{s}(z), z \in \mathbb{D}$, that is,

$$
\mathcal{P}\left(\mathfrak{q}_{s}\right)=\left\{p(z)=1+p_{1} z+p_{2} z^{2}+\cdots, p(z) \prec \mathfrak{q}_{s}(z), z \in \mathbb{D}\right\} \subset \mathcal{P} .
$$

Theorem 2.3 If $p \in \mathcal{P}\left(\mathfrak{q}_{s}\right)$, then

$$
\Re p(z)>\frac{1}{2^{s}}, \quad|\operatorname{Arg}\{p(z)\}|<\frac{\pi}{2} s \quad(z \in \mathbb{D}),
$$

and

$$
\left|p^{1 / s}(z)-1\right|<|p(z)|^{1 / s} \quad(z \in \mathbb{D})
$$

or

$$
\left|p^{1 /(2 s)}(z)+1\right|-\left|p^{1 /(2 s)}(z)-1\right|>\sqrt{2} \quad \text { and } \quad \Re p^{1 / s}(z)>\frac{1}{2} \quad(z \in \mathbb{D}) .
$$

Conversely, if $p$ is analytic with $p(0)=1,|\operatorname{Arg}\{p(z)\}|<(\pi s) / 2$ and $p$ satisfies (2.4) (or one of the conditions of (2.5)), then $p \prec \mathfrak{q}_{s}$ in $\mathbb{D}$.

Proof The subordination $p \prec \mathfrak{q}_{s}$ with $p(0)=\mathfrak{q}_{s}(0)$, and the geometric properties of $\mathfrak{q}_{s}(\mathbb{D})$ from Sect. 1, yield (2.3). We next claim that (2.4) is satisfied. Repeated application of the properties of subordination $p \prec \mathfrak{q}_{s}$ shows that there exists $\omega \in \mathcal{B}$, such that

$$
p(z)=\frac{1}{(1-\omega(z))^{s}} \quad \text { or } \quad p^{1 / s}(z)=\frac{1}{1-\omega(z)} \quad(z \in \mathbb{D})
$$

which is equivalent to

$$
\omega(z)=\frac{p^{1 / s}(z)-1}{p^{1 / s}(z)}, \quad|\omega(z)|<1 \quad(z \in \mathbb{D}),
$$

and the assertion (2.4) now follows. Squaring (2.4) we may rewrite it as

$$
\left(p^{1 / s}(z)-1\right)\left(\overline{p^{1 / s}(z)}-1\right)<\left|p^{1 / s}(z)\right|^{2}
$$

or

$$
\left|p^{1 / s}(z)\right|^{2}-p^{1 / s}(z)-\overline{p^{1 / s}(z)}+1<\left|p^{1 / s}(z)\right|^{2}
$$

which is nothing but the second relation of (2.5). For the proof of the first relation of (2.5) we rewrite (2.4) as

$$
2+2\left|p^{1 / s}(z)\right|-2\left|p^{1 / s}(z)-1\right|>2,
$$

or equivalently

$$
\left|p^{1 /(2 s)}(z)+1\right|^{2}+\left|p^{1 /(2 s)}(z)-1\right|^{2}-2\left|p^{\frac{1}{s}}(z)-1\right|>2,
$$


which immediately becomes

$$
\left(\left|p^{1 /(2 s)}(z)+1\right|-\left|p^{1 /(2 s)}(z)-1\right|\right)^{2}>2,
$$

and, from this, the desired conclusion follows.

Conversely, if for $p$ is analytic in $\mathbb{D}$ with $p(0)=1$ and the condition (2.4) (or one of the conditions of (2.5)) is satisfied, then we easily show that $p=\rho \mathrm{e}^{\mathrm{i} \theta}$ lies in a domain $\mathbb{H}(s)=$ $\mathfrak{q}_{s}(\mathbb{D})$, which completes the proof.

Theorem 2.4 If $p \in \mathcal{P}$ satisfies

$$
\Re\left\{\frac{z p^{\prime}(z)}{p(z)}\right\}>-\frac{s}{2} \quad(z \in \mathbb{D})
$$

then

$$
p(z) \prec \mathfrak{q}_{s}(z) \quad(z \in \mathbb{D}) .
$$

Proof Suppose, on the contrary, that $p(z) \nprec \mathfrak{q}_{s}(z)$ on $\mathbb{D}$. Then, by $p(0)=\mathfrak{q}_{s}(0)=1$, and by Lemma 1.1, there exist $z_{0} \in \mathbb{D}$ and $\zeta_{0} \in \partial \mathbb{D}\left(\zeta_{0} \neq 1\right)$ such that

$$
p\left(z_{0}\right)=\mathfrak{q}_{s}\left(\zeta_{0}\right), \quad z_{0} p^{\prime}\left(z_{0}\right)=m \zeta_{0} \mathfrak{q}_{s}^{\prime}\left(\zeta_{0}\right) \quad \text { with } m \geq 1
$$

Hence

$$
\Re\left\{\frac{z_{0} p^{\prime}\left(z_{0}\right)}{p\left(z_{0}\right)}\right\}=\Re\left\{\frac{m \zeta_{0} \mathfrak{q}_{s}^{\prime}\left(\zeta_{0}\right)}{\mathfrak{q}_{s}\left(\zeta_{0}\right)}\right\}=m s \Re\left\{\frac{\zeta_{0}}{1-\zeta_{0}}\right\}=-\frac{m s}{2} \leq-\frac{s}{2},
$$

which contradicts the assumption (2.6), and therefore $p \prec \mathfrak{q}_{s}$ on $\mathbb{D}$.

\section{The classes $\mathcal{S} \mathcal{T}_{\text {hpl }}(s), \mathcal{C} \mathcal{V}_{\text {hpl }}(s)$}

In this section we introduce and examine the notion of starlikeness and convexity related to the domains bounded by the hyperbolas $H(s)$. We indicate how the properties of the domains $\mathbb{H}(s)$ and class $\mathcal{P}\left(\mathfrak{q}_{s}\right)$ influence on the properties of a newly introduced classes.

Definition 3.1 Let $\mathcal{S} \mathcal{T}_{\text {hpl }}(s)$ denote the subfamily of $\mathcal{S}$ consisting of the functions $f$ satisfying the condition

$$
\frac{z f^{\prime}(z)}{f(z)} \prec \mathfrak{q}_{s}(z) \quad(z \in \mathbb{D}),
$$

and let $\mathcal{C} \mathcal{V}_{\text {hpl }}(s)$ be a class of analytic functions $f$ such that

$$
1+\frac{z f^{\prime \prime}(z)}{f^{\prime}(z)} \prec \mathfrak{q}_{s}(z) \quad(z \in \mathbb{D}) .
$$

Geometrically, the condition (3.1) and (3.2) means that the expression $z f^{\prime}(z) / f(z)$ (or $1+z f^{\prime \prime}(z) / f^{\prime}(z)$, resp. $)$ lies in a domain $\mathbb{H}(s)$. Since $\mathbb{H}(s)=\mathfrak{q}_{s}(\mathbb{D})$ is contained in a right halfplane, we deduce that $\mathcal{S} \mathcal{T}_{\text {hpl }}(s)$ is a proper subset of a class of a starlike functions $\mathcal{S T}$ (and 
$\mathcal{C} \mathcal{V}_{\text {hpl }}(s) \subset \mathcal{C V}$, resp.). Furthermore, the properties of $\mathbb{H}(s)$ considered in Sect. 1, yield

$$
\begin{aligned}
& \mathcal{S} \mathcal{T}_{\text {hpl }}(s) \subset \mathcal{S} \mathcal{T}(\alpha) \quad \text { and } \quad \mathcal{C} \mathcal{V}_{\text {hpl }}(s) \subset \mathcal{C V}(\alpha) \quad \text { for } 0 \leq \alpha \leq 2^{-s}, 0<s \leq 1, \\
& \mathcal{S} \mathcal{T}_{\text {hpl }}(s) \subset \mathcal{S} \mathcal{T}_{\gamma} \quad \text { and } \quad \mathcal{C} \mathcal{V}_{\text {hpl }}(s) \subset \mathcal{C} \mathcal{V}_{\gamma} \quad \text { for } 0<s \leq \gamma \leq 1
\end{aligned}
$$

The above inclusions additionally give

$$
\mathcal{S} \mathcal{T}_{\text {hpl }}(s) \subset \mathcal{S} \mathcal{T}_{s} \cap \mathcal{S} \mathcal{T}\left(1 / 2^{s}\right), \quad \mathcal{C} \mathcal{V}_{\text {hpl }}(s) \subset \mathcal{C} \mathcal{V}_{s} \cap \mathcal{C} \mathcal{V}\left(1 / 2^{s}\right), \quad 0<s \leq 1
$$

Also, geometric properties of $\Omega_{k}$ and $\mathbb{H}(s)$ given by (1.1), (1.2), (1.3) and also (1.4) imply

$$
\begin{aligned}
& \mathcal{S} \mathcal{T}_{\text {hpl }}(s) \subset k-\mathcal{S T} \text { and } \quad \mathcal{C} \mathcal{V}_{\text {hpl }}(s) \subset k-\mathcal{C V} \text { for } 0 \leq k \leq 2^{-s}, 0<s \leq 1, \\
& k-\mathcal{S} \mathcal{T} \subset \mathcal{S} \mathcal{T}_{\text {hpl }}(s) \text { and } \quad k-\mathcal{C V} \subset \mathcal{C} \mathcal{V}_{\text {hpl }}(s) \quad \text { for } k \geq 2^{s}-1,0<s \leq 1, \\
& k-\mathcal{S} \mathcal{T} \cap \mathcal{S} \mathcal{T}_{\text {hpl }}(s) \neq \emptyset \text { and } \quad k-\mathcal{C V} \cap \mathcal{C} \mathcal{V}_{\text {hpl }}(s) \neq \emptyset \text { for } 2^{-s}<k<2^{s}-1,0<s \leq 1
\end{aligned}
$$

Moreover

$$
k-\mathcal{S} \mathcal{T} \not \subset \mathcal{S} \mathcal{T}_{\text {hpl }}(s) \quad \text { and } \quad \mathcal{S} \mathcal{T}_{\text {hpl }}(s) \not \subset k-\mathcal{S T} \quad \text { for } 2^{-s}<k<2^{s}-1,0<s \leq 1 \text {, }
$$

and

$$
k-\mathcal{C V} \not \subset \mathcal{C} \mathcal{V}_{\text {hpl }}(s) \text { and } \quad \mathcal{C} \mathcal{V}_{\text {hpl }}(s) \not \subset k-\mathcal{C} \mathcal{V} \text { for } 2^{-s}<k<2^{s}-1,0<s \leq 1
$$

Since $\mathfrak{q}_{s}$ is the extremal function in $\mathcal{P}\left(\mathfrak{q}_{s}\right)$, the obvious integral representation of $\mathcal{S} \mathcal{T}_{\text {hpl }}(s)$ and $\mathcal{C} \mathcal{V}_{\text {hpl }}(s)$ immediately follows.

Theorem 3.2 A function $f$ is in the class $\mathcal{S} \mathcal{T}_{\mathrm{hpl}}(s)$ if, and only if there exists $p$ such that $p \prec \mathfrak{q}_{s}$, and

$$
f(z)=z \exp \left(\int_{0}^{z} \frac{p(t)-1}{t} \mathrm{~d} t\right) .
$$

Theorem 3.3 A function $f$ is in the class $\mathcal{C} \mathcal{V}_{\mathrm{hpl}}(s)$ if, and only if there exists $p$ such that $p \prec \mathfrak{q}_{s}$, and

$$
f(z)=\int_{0}^{z} \exp \left(\int_{0}^{x} \frac{p(t)-1}{t} \mathrm{~d} t\right) \mathrm{d} x .
$$

Suppose that $\Phi_{s, n} \in \mathcal{S} \mathcal{T}_{\text {hpl }}(s)$ is such that

$$
\frac{z \Phi_{s, n}^{\prime}(z)}{\Phi_{s, n}(z)}=\frac{1}{\left(1-z^{n}\right)^{s}} \quad(z \in \mathbb{D}, n=1,2, \ldots) .
$$

Then the functions $\Phi_{s, n}(z)$ are of the form

$$
\begin{aligned}
\Phi_{s, n}(z)= & z \exp \left(\int_{0}^{z} \frac{\mathfrak{q}_{s}\left(t^{n}\right)-1}{t} \mathrm{~d} t\right)=z+\frac{s}{n} z^{n+1}+\frac{(n+2) s^{2}+n s}{4 n^{2}} z^{2 n+1} \\
& +\frac{4 n^{2} s+\left(9 n+6 n^{2}\right) s^{2}+\left(2 n^{2}+9 n+6\right) s^{3}}{36 n^{3}} z^{3 n+1} \cdots
\end{aligned}
$$


and these are extremal functions for different problems in the class $\mathcal{S} \mathcal{T}_{\text {hpl }}(s)$. For instance

$$
\begin{aligned}
\Phi_{s}(z) & =\Phi_{s, 1}(z)=z \exp \left(\int_{0}^{z} \frac{\mathfrak{q}_{s}(t)-1}{t} \mathrm{~d} t\right) \\
& =z+s z^{2}+\frac{3 s^{2}+s}{4} z^{3}+\frac{17 s^{3}+15 s^{2}+4 s}{36} z^{4} \cdots \quad(z \in \mathbb{D}) .
\end{aligned}
$$

The special case $s=1 / 2$ gives

$$
\begin{aligned}
\Phi_{1 / 2, n}(z) & =z \exp \left(\int_{0}^{z} \frac{\mathfrak{q}_{1 / 2}\left(t^{n}\right)-1}{t} \mathrm{~d} t\right)=z\left(\frac{4}{\left(1+\sqrt{1-z^{n}}\right)^{2}}\right)^{1 / n}, \\
& =z+\frac{1}{2 n} z^{n+1}+\frac{7-2 n}{16 n^{2}} z^{2 n+1}+\cdots
\end{aligned}
$$

and

$$
\begin{aligned}
\Phi_{1 / 2}(z) & =\Phi_{1 / 2,1}(z)=z \exp \left(\int_{0}^{z} \frac{\mathfrak{q}_{1 / 2}(t)-1}{t} \mathrm{~d} t\right)=\frac{4 z}{(1+\sqrt{1-z})^{2}} \\
& =z+\frac{1}{2} z^{2}+\frac{5}{16} z^{3}+\cdots \quad(z \in \mathbb{D}) .
\end{aligned}
$$

Also, suppose that $K_{s, n} \in \mathcal{C} \mathcal{V}_{\text {hpl }}(s)$ is such that

$$
1+\frac{z K_{s, n}^{\prime \prime}(z)}{K_{s, n}^{\prime}(z)}=\frac{1}{\left(1-z^{n}\right)^{s}} \quad(z \in \mathbb{D}, n=1,2, \ldots)
$$

Then the functions $K_{s, n}(z)$ are of the form

$$
\begin{aligned}
K_{s, n}(z)= & \int_{0}^{z} \exp \left(\int_{0}^{w} \frac{\mathfrak{q}_{s}\left(t^{n}\right)-1}{t} \mathrm{~d} t\right) \mathrm{d} w \\
= & z+\frac{s}{n(n+1)} z^{n+1}+\frac{(n+2) s^{2}+n s}{4 n^{2}(2 n+1)} z^{2 n+1} \\
& +\frac{4 n^{2} s+\left(9 n+6 n^{2}\right) s^{2}+\left(2 n^{2}+9 n+6\right) s^{3}}{36 n^{3}(3 n+1)} z^{3 n+1} \cdots,
\end{aligned}
$$

and the $K_{s, n}$ are extremal functions for various problems in the class $\mathcal{C} \mathcal{V}_{\text {hpl }}(s)$. The most interesting special cases are for $n=1$

$$
\begin{aligned}
K_{s}(z) & =K_{s, 1}(z)=\int_{0}^{z} \exp \left(\int_{0}^{w} \frac{\mathfrak{q}_{s}(t)-1}{t} \mathrm{~d} t\right) \mathrm{d} w=\int_{0}^{z} \frac{\Phi_{s}(w)}{w} \mathrm{~d} w \\
& =z+\frac{s}{2} z^{2}+\frac{3 s^{2}+s}{12} z^{3}+\frac{17 s^{3}+15 s^{2}+4 s}{144} z^{4} \cdots \quad(z \in \mathbb{D})
\end{aligned}
$$

and for $s=1 / 2, n=1$

$$
\begin{aligned}
K_{1 / 2}(z)=K_{1 / 2,1}(z) & =\int_{0}^{z} \frac{\Phi_{1 / 2}(w)}{w} \mathrm{~d} w \\
& =8 \log \left(\frac{2}{1+\sqrt{1-z}}\right)+4\left(\frac{\sqrt{1-z}-1}{\sqrt{1-z}+1}\right) \\
& =z+\frac{1}{4} z^{2}+\frac{5}{48} z^{3}+\frac{7}{128} z^{4}+\frac{21}{640} z^{5}+\cdots(z \in \mathbb{D}) .
\end{aligned}
$$


From Theorems 3.2 and 3.3 we obtain the following corollary.

Corollary 3.4 Iff $\in \mathcal{C V}(1-s / 2)$, then $f^{\prime} \prec \mathfrak{q}_{s}$ in $\mathbb{D}$. Thus $f$ is univalent in $\mathbb{D}$. The functions $H$ and $G$ defined by

$$
H(z)=z \exp \left(\int_{0}^{z} \frac{f^{\prime}(t)-1}{t} \mathrm{~d} t\right), \quad G(z)=\int_{0}^{z} \exp \left(\int_{0}^{x} \frac{f^{\prime}(t)-1}{t} \mathrm{~d} t\right) \mathrm{d} x,
$$

belong to $\mathcal{S} \mathcal{T}_{\mathrm{hpl}}(s)$ and $\mathcal{C} \mathcal{V}_{\mathrm{hpl}}(s)$, respectively.

Examples Defined classes $\mathcal{S} \mathcal{T}_{\text {hpl }}(s)$ and $\mathcal{C} \mathcal{V}_{\text {hpl }}(s)$ are nonempty. The integral representation given in Theorems 3.2 and 3.3 provides various examples of functions of those classes. For example, if $q_{1}(z)=1+B_{1} z$ with $0<B_{1}<1-2^{-s}$, then $q_{1} \prec \mathfrak{q}_{s}$. Hence $f_{1}(z)=z \exp \left(B_{1} z\right) \in$ $\mathcal{S} \mathcal{T}_{\text {hpl }}(s)$, and $g_{1}(z)=\left(e^{B_{1} z}-1\right) / B_{1} \in \mathcal{C} \mathcal{V}_{\text {hpl }}(s)\left(\right.$ for $\left.0<B_{1}<1-2^{-s}\right)$, respectively. Since

$$
f_{2}(z)=\frac{z}{1-B_{2} z}, \quad f_{3}(z)=\frac{z}{\left(1-B_{3} z\right)^{2}}
$$

for $0<\left|B_{2}\right| \leq s /(2-s), 0<\left|B_{3}\right| \leq s /(4-s)$ are starlike of order $(1-s / 2)$, applying Corollary 3.4 we see that the appropriate functions

$$
H_{2}(z)=\frac{z}{1-B_{2} z}, \quad H_{3}(z)=\frac{z}{1-B_{3} z} \exp \left(\frac{B_{3} z}{1-B_{3} z}\right)
$$

belong to the class $\mathcal{S} \mathcal{T}_{\text {hpl }}(s)$, and the corresponding functions will be elements of $\mathcal{C} \mathcal{V}_{\text {hpl }}(s)$.

Theorem 3.5 The function $\Phi_{s, n}(z)$, defined by (3.4), is normalized, univalent and convex in one direction in $\mathbb{D}$ for $n=1,2, \ldots,\left\lfloor\left(2^{1-s}+1\right) / s\right\rfloor$, and convex for $n=1,2, \ldots,\left\lfloor 2^{1-s} / s\right\rfloor$, where $\lfloor\cdot\rfloor$ is the floor function.

Proof It is a simple matter to check that $\Phi_{s, n}(z)$ is normalized by $\Phi_{s, n}(0)=\Phi_{s, n}^{\prime}(0)-1=0$. By a definition (3.3) of $\Phi_{s, n}(z)$, we have

$$
\frac{\Phi_{s, n}^{\prime}(z)}{\Phi_{s, n}(z)}=\frac{1}{z\left(1-z^{n}\right)^{s}}
$$

Hence

$$
\mathfrak{R}\left(1+\frac{z \Phi_{s, n}^{\prime \prime}(z)}{\Phi_{s, n}^{\prime}(z)}\right)=\mathfrak{R}\left(n s \frac{z^{n}}{1-z^{n}}+\frac{1}{\left(1-z^{n}\right)^{s}}\right),
$$

and from the above we see that

$$
\Re\left(1+\frac{z \Phi_{s, n}^{\prime \prime}(z)}{\Phi_{s, n}^{\prime}(z)}\right)>2^{-s}-\frac{n s}{2}
$$

In order to get univalence, it is convenient to use the result by Umezawa [12], with the requirement $\Re\left(1+z h^{\prime \prime}(z) / h^{\prime}(z)\right)>-1 / 2$ for univalence of $h$ in $\mathbb{D}$. That condition holds if

$$
2^{-s}-\frac{n s}{2} \geq-\frac{1}{2}
$$


which is satisfied for $n=1,2, \ldots,\left\lfloor\left(2^{1-s}+1\right) / s\right\rfloor$. We recall that the same condition gives the convexity in one direction. Furthermore, $\Phi_{s, n}(z)$ is convex in $\mathbb{D}$ if $\Re\left(1+z \Phi_{s, n}^{\prime \prime}(z) / \Phi_{s, n}^{\prime}(z)\right)>0$ holds for $n \leq 2^{1-s} / s$, i.e. for $n=1,2, \ldots,\left\lfloor 2^{1-s} / s\right\rfloor$.

We have the following from the results in [7], and Lemma 2.1.

Corollary 3.6 If $f \in \mathcal{S T}_{\mathrm{hpl}}(s)$ and $|z|=r<1$, then

$$
\begin{aligned}
& -\Phi_{s}(-r) \leq|f(z)| \leq \Phi_{s}(r), \\
& \Phi_{s}^{\prime}(-r) \leq\left|f^{\prime}(z)\right| \leq \Phi_{s}^{\prime}(r), \\
& |\operatorname{Arg}\{f(z) / z\}| \leq \max _{|z|=r} \operatorname{Arg}\left\{\Phi_{s}(z) / z\right\}, \\
& f(z) / z \prec \Phi_{s}(z) / z \quad(z \in \mathbb{D}) .
\end{aligned}
$$

Equality holds for some $z_{0} \neq 0$ if and only iff is a rotation of $\Phi_{s}$, where $\Phi_{s}$ is given by (3.5). Also, iff $\in \mathcal{S} \mathcal{T}_{\text {hpl }}(s)$, then either $f$ is a rotation of $\Phi_{s}$ or

$$
\left\{w \in \mathbb{C}:|w| \leq-\Phi_{s}(-1)\right\} \subset f(\mathbb{D}) .
$$

Here $-\Phi_{s}(-1)$ is understood to be the limit of $-\Phi_{s}(-r)$ as $r$ tends to 1 .

Similar results hold for functions of $\mathcal{C} \mathcal{V}_{\text {hpl }}(s)$.

Corollary 3.7 If $f \in \mathcal{C} \mathcal{V}_{\mathrm{hpl}}(s)$ and $|z|=r<1$, then

$$
\begin{aligned}
& -K_{s}(-r) \leq|f(z)| \leq K_{s}(r), \\
& K_{s}^{\prime}(-r) \leq\left|f^{\prime}(z)\right| \leq K_{s}^{\prime}(r), \\
& \left|\operatorname{Arg}\left\{f^{\prime}(z)\right\}\right| \leq \max _{|z|=r} \operatorname{Arg}\left\{K_{s}^{\prime}(z)\right\}, \\
& f^{\prime}(z) \prec K_{s}^{\prime}(z) \quad(z \in \mathbb{D}) .
\end{aligned}
$$

Equality holds for some $z_{0} \neq 0$ if and only iff is a rotation of $K_{s}$, where $K_{s}$ is given by (3.7). Also, if $f \in \mathcal{C} \mathcal{V}_{\mathrm{hpl}}(s)$, then either $f$ is a rotation of $K_{s}$ or

$$
\left\{w \in \mathbb{C}:|w| \leq-K_{s}(-1)\right\} \subset f(\mathbb{D}) .
$$

Here $-K_{s}(-1)$ is understood to be the limit of $-K_{s}(-r)$ as $r$ tends to 1 .

Theorem 3.8 Let $r_{0}$ denote the positive root of the equation

$$
\frac{1}{(1+r)^{s}}=\frac{r s}{1-r} \quad(0 \leq r<1) .
$$

If $\in \mathcal{S} \mathcal{T}_{\mathrm{hpl}}(s)$, then $f$ is convex in the disk $|z|<r_{0}$. 
Proof Let $f \in \mathcal{S} \mathcal{T}_{\text {hpl }}(s)$. Then from Definition 3.1 we obtain

$$
\frac{z f^{\prime}(z)}{f(z)}=\frac{1}{(1-w(z))^{s}} \quad(z \in \mathbb{D})
$$

where $w \in \mathcal{B}$. Application of Lemma 2.1 and the well-known inequality for Schwarz functions $\left|w^{\prime}(z)\right| \leq\left(1-|w(z)|^{2}\right) /\left(1-|z|^{2}\right)$ gives

$$
\begin{aligned}
\Re\left(1+\frac{z f^{\prime \prime}(z)}{f^{\prime}(z)}\right) & \geq \Re \frac{1}{(1-w(z))^{s}}-s|z| \frac{1-|w(z)|^{2}}{[1-|w(z)|]\left[1-|z|^{2}\right]} \\
& \geq \frac{1}{(1+|z|)^{s}}-\frac{s|z|}{1-|z|} .
\end{aligned}
$$

The function $\psi(r)=\frac{1}{(1+r)^{s}}-\frac{r s}{1-r}$, where $r=|z| \in[0,1)$ is decreasing in $[0,1)$ with $\psi(0)=1$ and $\psi\left(1^{-}\right)=-\infty$. Thus, there exists a unique $r_{0} \in(0,1)$ such that $\psi\left(r_{0}\right)=0$, and for $0 \leq r<$ $r_{0}$ we have $0<\psi(r) \leq 1$. This inequality is equivalent to

$$
\frac{1}{(1+r)^{s}}>\frac{r s}{1-r} \quad(r \in[0,1))
$$

which is satisfied for $|z|<r_{0}$, where $r_{0}$ is the only real positive root of (3.8).

Theorem 3.9 Iff $\in \mathcal{S} \mathcal{T}_{\text {hpl }}(s)$, then there exists $\alpha>1$ such that

$$
\Re\left\{1+\frac{z f^{\prime \prime}(z)}{f^{\prime}(z)}\right\}<\alpha \quad(|z|=r<1)
$$

Proof Let $f \in \mathcal{S} \mathcal{T}_{\text {hpl }}(s)$. Then, from Definition 3.1, we obtain

$$
\frac{z f^{\prime}(z)}{f(z)}=\frac{1}{(1-w(z))^{s}} \quad(z \in \mathbb{D}),
$$

where $w \in \mathcal{B}$. By Lemma 2.1 and the inequality for Schwarz functions it follows that

$$
\begin{aligned}
\Re\left(1+\frac{z f^{\prime \prime}(z)}{f^{\prime}(z)}\right) & \leq \Re \frac{1}{(1-w(z))^{s}}+s|z| \frac{1-|w(z)|^{2}}{[1-|w(z)|]\left[1-|z|^{2}\right]} \\
& \leq \frac{1}{(1-|z|)^{s}}+\frac{s|z|}{1-|z|} .
\end{aligned}
$$

The function $g(r)=\frac{1}{(1-r)^{s}}+\frac{r s}{1-r}$, where $r=|z| \in(0,1)$, is increasing in $(0,1)$ and $g(0)=1$. Thus for $z \in \mathbb{D}$ we have $g(|z|)>1$.

Acknowledgements

The authors thank the editor and the anonymous referees for constructive and pertinent suggestions.

Funding

This work was partially supported by the Centre for Innovation and Transfer of Natural Sciences and Engineering Knowledge, Faculty of Mathematics and Natural Sciences, University of Rzeszow. 
Competing interests

The authors declare that they have no competing interests.

\section{Authors' contributions}

Each of the authors contributed to each part of this study equally, all authors read and approved the final manuscript.

\section{Author details}

${ }^{1}$ University of Rzeszow, Rzeszów, Poland. ${ }^{2}$ Department of Mathematics, Payame Noor University (PNU), P.O. Box,

19395-3697, Tehran, Iran. ${ }^{3}$ Department of Mathematics, Faculty of Science, Urmia University, Urmia, Iran.

\section{Publisher's Note}

Springer Nature remains neutral with regard to jurisdictional claims in published maps and institutional affiliations.

Received: 26 March 2019 Accepted: 27 August 2019 Published online: 12 September 2019

\section{References}

1. Brannan, D.A., Kirwan, W.E.: On some classes of bounded univalent functions. J. Lond. Math. Soc. 2(1), 431-443 (1969)

2. Duren, P.L.: Univalent Functions. Grundlehren der Mathematischen Wissenschaften, vol. 259. Springer, New York (1983)

3. Goodman, A.W.: Univalent Functions, Vols. I and II. Mariner Publishing Company, Tampa (1983)

4. Kanas, S., Sugawa, T.: On conformal representations of the interior of an ellipse. Ann. Acad. Sci. Fenn., Math. 31, 329-348 (2006)

5. Kanas, S., Wisniowska, A.: Conic regions and k-uniform convexity. J. Comput. Appl. Math. 105, 327-336 (1999)

6. Kanas, S., Wisniowska, A.: Conic domains and starlike functions. Rev. Roum. Math. Pures Appl. 45(4), $647-658$ (2000)

7. Ma, W.C., Minda, D.: A unified treatment of some special classes of univalent functions. In: Li, Z., Ren, F., Yang, L., Zhang, S. (eds.) Proc. Int. Conf. on Complex Analysis, pp. 157-169. International Press, Cambridge (1994)

8. Miller, S.S., Mocanu, P.T.: Differential Subordinations: Theory and Applications. Series of Monographs and Textbooks in Pure and Applied Mathematics, vol. 225. Dekker, New York (2000)

9. Robertson, M.I.: On the theory of univalent functions. Appl. Math., 374-408 (1936)

10. Royster, W.C.: On the univalence of a certain integral. Mich. Math. J. 12(4), 385-387 (1965)

11. Stankiewicz, J.: Quelques problèmes extrèmaux dans les classes des fonctions $\alpha$-angulairement ètoilèes. Ann. Univ. Mariae Curie-Skłodowska, Sect. A 20, 59-75 (1966)

12. Umezawa, T.: Analytic functions convex in one direction. J. Math. Soc. Jpn. 4, 194-202 (1952)

\section{Submit your manuscript to a SpringerOpen ${ }^{\circ}$ journal and benefit from:}

- Convenient online submission

- Rigorous peer review

- Open access: articles freely available online

- High visibility within the field

- Retaining the copyright to your article

Submit your next manuscript at $\gg$ springeropen.com 